\title{
ESTUDO DA MOTILIDADE ESOFÁGICA APÓS LIGADURA ELÁSTICA ENDOSCÓPICA DAS VARIZES
}

Ana Cristina fontenele Soares, Dráusio Jefferson Morais, José Olympio Meirelles Santos, luiz Roberto lopes, Nelson Adami Andreollo*

Trabalho realizado no Centro de Diagnóstico de Doenças do Aparelho Digestivo da Faculdade de Ciências Médicas da Universidade Estadual de Campinas - Campinas SP

*Correspondência:

Rua Francisco Humberto

Zuppi, 1234, Cidade

Universitária, Campinas, SP

Cep: 13.083-350,

nandreollo@terra.com.br

\section{RESUMO}

OBjetivos. Estudar a motilidade esofágica de doentes cirróticos antes e após a ligadura elástica endoscópica das varizes. Métodos. Vinte e quatro portadores de cirrose hepática atendidos no Gastrocentro - UNICAMP, no programa de ligadura elástica para tratamento de varizes, foram estudados (média de idade de 49,5 anos, sendo 19 masculinos e 5 femininos). Os critérios de inclusão foram hepatopatia crônica e varizes esofágicas com alto risco de sangramento. Inicialmente foram realizados endoscopia digestiva alta e manometria esofágica em todos os doentes. A seguir, foram submetidos a sessões de ligadura elástica (o número médio foi de $3,4 \pm 2,1$ ), em regime ambulatorial, com intervalo de duas a quatro semanas. A manometria foi repetida quatro semanas após a erradicação das varizes. Os parâmetros estudados foram a amplitude, a duração, a velocidade de propagação das ondas contráteis e o peristaltismo.

Resultados. A análise do tônus do EIE não mostrou diferença entre pré e pós-ligadura elástica. Em dez casos (4I,6\%), ocorreu alteração na motilidade, e a amplitude das ondas de deglutição elevou-se no exame pré de $70,7 \mathrm{mmHg}(52,3$ e $108,4)$ para $89,7 \mathrm{mmHg}(69,9$ e I22,8) no pós $(p=0,004-p<0,05)$, e a duração aumentou de 3,55 seg $\pm 0,58$ no pré para 3,90 seg $\pm 0,72$ no pós $(p=0,02-p<0,05)$. A velocidade das ondas não diferiu entre o exame pré $3,43 \pm 0,97 \mathrm{~cm} / \mathrm{seg}$ e pós $3,61 \pm 0,99 \mathrm{~cm} / \mathrm{seg}(p=0,15-p>0,05)$.

Conclusōes. A análise final mostra que ocorreu alteraçãa de motilidade esofágica após ligadura elástica das varizes esofágicas, caracterizada por aumento na amplitude e duração das ondas contráteis.

UnItermos: Varizes esofágicas. Endoscopia digestiva. Cirrose hepática. Motilidade esofágica.

\section{INTRODUÇÃO}

A hemorragia digestiva alta (HDA) secundária à hipertensão portal é uma das principais complicações observadas em doentes hepatopatas crônicos. Dois procedimentos endoscópicos são indispensáveis para o seu tratamento adequado: a escleroterapia e a ligadura elástica das varizes esôfago-gástricas ${ }^{\prime, 2}$.

A escleroterapia é um procedimento de baixo custo, realizado na maioria dos serviços de endoscopia, podendo ser utilizado não apenas nos episódios de hemorragia digestiva, como na prevenção e no controle das varizes esofágicas²,3. Entretanto, vários estudos já demonstraram que os agentes esclerosantes aplicados durante a escleroterapia causam alterações macro e microscocópicas na parede do esôfago e conseqüentes efeitos adversos na motilidade esofágica ${ }^{4-8}$. Estudo prévio realizado em nosso serviço comparando a manometria esofágica antes e após a escleroterapia mostrou modificações no peristaltismo esofágico, secundário à seqüela da fibrose que ocorre na mucosa e submucosa do órgão6.

A ligadura elástica é um método mais recente que a escleroterapia. É considerado mais seguro no tratamento das varizes esofágicas e de custo acessível para ser utilizado em larga esca-

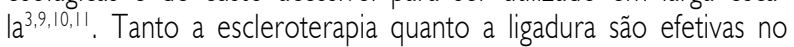
tratamento das varizes esofágicas, entretanto, a incidência de complicações e a recorrência de sangramento são menos freqüentes após a ligadura ${ }^{12}$. Steigmann ${ }^{9}$ e colaboradores relataram que a ligadura elástica causa uma resposta inflamatória na submucosa que pode persistir por três semanas pós ligadura, podendo causar alteração na motilidade esofágica. Entretanto, outros poucos estudos foram realizados avaliando o efeito da ligadura elástica na motilidade esofágica e os resultados são contraditórios ${ }^{8,10,12,13}$.

O objetivo deste estudo é avaliar os efeitos da ligadura elástica de varizes na motilidade do esôfago, por meio da manometria antes e após a ligadura em doentes cirróticos.

\section{Métodos}

Foram incluídos no estudo 24 doentes com cirrose hepática, com alto risco de sangramento, atendidos no Gastrocentro - Unicamp, no programa de ligadura elástica para tratamento de varizes esofágicas, no período de dezembro de 1998 a dezembro de 2000. A média da idade do grupo ( \pm DP) foi igual a $49,5( \pm 13,8)$ anos, sendo 19 do sexo masculino e 5 do sexo feminino. Seis pacientes apresentaram HDA antes da inclusão no estudo. Quanto à etiologia da cirrose hepática, sete doentes eram etilistas crônicos (29,3\%), cinco eram portadores de vírus $\mathrm{B}(20,5 \%)$, I I do vírus C $(45,9 \%)$ e um de etiologia desconhecida (4,3\%).

A classificação de Child-Pugh ${ }^{2}$ mostrou que I I doentes eram classe A (45,9\%), nove eram classe B $(37,5 \%)$ e dois classe C (8,6\%). $O$ número médio de ligaduras elásticas foi $3,4 \pm 2,1$, sendo que o número de anéis elásticos por doente foi 14,2 7,6. 
SoARES ACF ET AL.

O presente estudo recebeu prévia aprovação do Comitê de Ética em Pesquisa, tendo sido obtidos de todos os pacientes o consentimento informado.

\section{Endoscopia digestiva alta e ligadura elástrica}

A endoscopia digestiva alta foi inicialmente indicada para diagnóstico e classificação das varizes esofágicas, seguindo a classificação da Japanese Research Societyfor Portal Hypertension². As sessões de ligadura elástica foram realizadas em regime ambulatorial, com intervalo de duas a quatro semanas. O procedimento foi realizado empregando-se equipamento de endoscopia digestiva flexível (Olympus) e o estojo de ligadura elástica Shooter-Saeed Multi-band ligator (Wilson-Cook U.S.A.). Após o término das sessões, o exame foi repetido para controle de varizes residuais.

\section{Manometria esofágica}

O estudo de motilidade esofágica foi realizado em duas ocasiões, antes da ligadura elástica e quatro semanas após a erradicação das varizes. Os doentes foram orientados a permanecer em jejum de oito horas, suspendendo o uso de quaisquer medicações procinéticas, ou outras medicações que pudessem eventualmente interferir na motilidade esofágica nas últimas 72 horas. $O$ exame foi realizado utilizando polígrafo computadorizado de oito canais da marca Synectics Medical (Stockholm, Sweden), modelo PC Polygraf HR, conectado a microcomputador com software para análise e interpretação dos dados. Além disso, foi utilizado cateter ECM8-R, com oito canais de registro, com diâmetro interno de I,5 $\mathrm{mm}$ e orifícios laterais de I $\mathrm{mm}$, sendo respectivamente dispostos em quatro canais radiais com distâncias de $90^{\circ}$ entre si na extremidade e outros quatro canais distantes $5 \mathrm{~cm}$ um do outro. Os canais foram infundidos com água, utilizando bomba de infusão capilar pneumohidráulica à pressão de nitrogênio, da marca $/ S$ Biomedicals (Ventura, California - USA), com fluxo de 0,6m/ $/ \mathrm{min} /$ canal.

Os cateteres foram introduzidos via nasal até o estômago, após aplicação tópica de xilocaína gel. O esfíncter inferior do esôfago (EIE) foi estudado com a técnica de tração lenta, ou seja, tracionando-se a sonda $\mathrm{Icm}$ por vez. O comprimento e a pressão do EIE foram aferidos utilizando-se a pressão intragástrica como referência zero. A distância do ponto zero da pressão intragástrica à posição do ponto de inversão da pressão (PI) foi medida como o comprimento do EIE. A pressão do EIE foi calculada como a diferença entre a pressão intragástrica e a pressão máxima do EIE durante a expiração, e considerada pela média aritmética simples das pressões obtidas em cada um dos quatro canais na extremidade do cateter, expressa em $\mathrm{mmHg}$.

O corpo do esôfago foi estudado nas porções inferior, média e superior, ou seja, 5,10 e $15 \mathrm{~cm}$ acima do EIE por meio de dez deglutições de saliva ou pequenas quantidades de água $(5 \mathrm{ml})$, e a amplitude das ondas esofágicas de deglutição foi analisada e comparada com a de indivíduos normais.

As ondas de deglutição foram analisadas levando-se em consideração os seguintes parâmetros: amplitude, morfologia, velocidade, duração e sincronismo. A morfologia, o sincronismo e a duração foram considerados após a análise visual de cada onda. A amplitude foi expressa em $\mathrm{mmHg}$, tendo como parâmetro principal as ondas contráteis do terço distal do esôfago. A duração foi expressa em segundos (seg). A velocidade de propagação da onda de deglutiçã̃o foi expressa em centímetros por segundo (cm/seg) e foi calculada mediante o intervalo tempo que a mesma levou para percorrer a distância entre o primeiro e o último canal de registro do cateter.

\section{Análise estatística}

Foram utilizados os testes t de Student e Mann-Whitney, sendo que o nível de rejeição para hipótese de nulidade foi fixado em um valor de $p$ igual ou menor do que $0,05(5 \%)(p<0,05)$.

\section{Resultados}

Os resultados de manometria pré e pós-ligadura elástica, analisando a extensão e a pressão do esfincter inferior do esôfago, a amplitude, a duração e a velocidade de propagação da onda de deglutição e o peristaltismo esofágico são mostrados na Tabela I.

$A$ análise EIE não mostrou diferença estatisticamente significante em relação à pressão e sua extensão no exame pré e pós-ligadura, bem como o seu relaxamento foi considerado normal em todos os casos $(p>0,05)$. Portanto, o EIE não sofreu alterações decorrentes da ligadura elástica.

$\mathrm{Na}$ comparação entre as medianas (percentis 25 e 75 entre parênteses) da amplitude das ondas esofágicas pré e pós-ligadura, foi observado um aumento pós-ligadura elástica, quando comparado com - exame pré-ligadura, sendo esta diferença estatisticamente significante $(p<0,05)$. A duração das ondas não diferiu no exame pré e pósligadura elástica $(p>0,05)$. Por outro lado, a velocidade de propagação das ondas de deglutição $(\mathrm{cm} / \mathrm{seg})$ nos exames pós-ligadura sofreu aumento estatisticamente significativo em comparação aos exames pré-ligadura $(p>0,05)$.

Quanto ao padrão de motilidade esofágica pós-ligadura, foi observado que em dez pacientes houve modificação do padrão de motilidade esofágica em comparaçãa ao estudo pré-ligadura, principalmente relacionado ao corpo esofágico. $\mathrm{Na}$ análise do peristaltismo das ondas contráteis esofágicas, apenas um paciente apresentou onda aperistática no exame pré-ligadura e este se manteve inalterado no exame pós-ligadura. Além disso, um paciente que apresentava ondas peristálticas no exame inicial, após a ligadura elástica apresentou $26 \%$ de ondas aperistálticas.

Dos 24 doentes incluídos no presente estudo, 14 (58,3\%) tinham ascite durante a realização do exame. Como a presença de ascite pode interferir na pressão do EIE, foram analisadas comparativamente as pressões do EIE entre os doentes com e sem ascite. $\mathrm{A}$ análise não mostrou diferença estatisticamente significante: sem ascite - 10,5 (9,2 e 15,4) $\mathrm{mmHg}$, e com ascite I2,7 (I0 e I5,4) $\mathrm{mmHg}(p=0,558)$.

No seguimento clínico (até dezembro/2003), cinco doentes necessitaram de nova sessão de ligadura elástica e outros três foram também submetidos à escleroterapia endoscópica concomitante das varizes. Seis pacientes foram a óbito por complicações decorrentes da hepatopatia crônica, sendo que dois foram vítimas de hemorragia digestiva alta; nove estão em acompanhamento clínico no Serviço de Hepatologia da Unicamp; três realizaram transplante hepático; e outros seis estão em lista de transplantes. Um doente abandonou o seguimento clínico. 


\begin{tabular}{|c|c|c|c|}
\hline & Pré- ligadura & Pós - ligadura & $\mathrm{p}$ \\
\hline \multicolumn{4}{|l|}{ Esfincter inferior do esôfago } \\
\hline $\begin{array}{ll} & \text { Extensão }(\mathrm{cm})^{\prime} \\
& \text { Pressão }(\mathrm{mmHg})^{2}\end{array}$ & $\begin{array}{c}3,85 \pm 0,87 \\
11,3\end{array}$ & $\begin{array}{c}3,75 \pm 0,80 \\
11,8\end{array}$ & 0,615 \\
\hline & $(9,27-15,3)$ & $(9,92-17,0)$ & 0,858 \\
\hline \multicolumn{4}{|l|}{ Corpo do esôfago } \\
\hline Amplitude $(\mathrm{mmHg})^{2}$ & $\begin{array}{c}70,7 \\
(52,3-108,4)\end{array}$ & $\begin{array}{c}89,7 \\
(69,9-122,8)\end{array}$ & $0,004 *$ \\
\hline Duração(seg) $)^{2}$ & $3,43 \pm 0,97$ & $3,61 \pm 0,99$ & 0,150 \\
\hline Velocidade $(\mathrm{cm} / \mathrm{seg})^{2}$ & $3,55 \pm 0,58$ & $3,90 \pm 0,58$ & $0,026 *$ \\
\hline \multicolumn{4}{|l|}{ Ondas esofágicas } \\
\hline $\begin{array}{l}\text { Peristáticas } \\
\text { Aperistálticas }\end{array}$ & $\begin{array}{c}23(91,7 \%) \\
1(8,3 \%)\end{array}$ & $\begin{array}{l}22(83,4 \%) \\
2(16,6 \%)\end{array}$ & \\
\hline
\end{tabular}

I. Teste t de Studant (seg - segundos; cm/seg - centímetros por segundo)

2. Teste de Mann Whitney * $p<0,05$

\section{Discussão}

A ligadura elástica é um procedimento mais seguro e efetivo que a escleroterapia para o tratamento das varizes esofágicas, já que os agentes esclerosantes podem causar alterações na parede do órgão e efeitos adversos na sua motilidade ${ }^{3,11,14,15}$. As alterações na motilidade esofágica após escleroterapia já foram estudadas por diversos autores, principalmente por meio da manometria esofágica. As principais alterações estão relacionadas com a morfologia, amplitude e duração das ondas contráteis. Estas alterações seriam, segundo alguns autores, secundárias a seqüelas de fibrose na parede do órgão5,6,7,10,12,14. Até o momento, poucos estudos foram realizados para avaliar as alterações da motilidade esofágica após ligadura elástica. Chen et al. ${ }^{10}$ estudaram 45 doentes com varizes esofágicas com alto risco de sangramento e realizaram manometria esofágica quatro a seis semanas após ligadura, concluindo que a $10 \mathrm{~cm}$ acima do EIE a amplitude das ondas de contração esofágica foi significativamente inferior em comparação ao grupo controle. Concluíram também que a porcentagem de ondas terciárias foi mais elevada após a ligadura, e que o comprimento do EIE não sofreu modificações quando comparado ao grupo controle. Estes resultados também foram confirmados por Kim et al. ${ }^{16} \mathrm{e}$ Passaretti et al. ${ }^{17}$, por outro lado, relataram que a presença de varizes residuais no terço inferior do esôfago após procedimentos endoscópicos pode diminuir a amplitude e duração das ondas peristálticas.

Goff et al. ${ }^{13}$ compararam dois grupos de cirróticos submetidos tanto à escleroterapia (oito doentes) como à ligadura elástica ( 12 doentes), e concluíram que as ondas de contração não diferiram quanto à amplitude, duração e velocidade de propagação nos dois grupos. Além disso, não registraram diferenças na pressão do ElE, porém o grupo submetido à escleroterapia apresentou porcentagem de relaxamento do EIE inferior ao grupo controle.

Avgerinos et al. ${ }^{18}$, analisando a pressão do EIE por meio de manometria esofágica imediatamente após a realização de esclero- terapia (oito doentes) e a ligadura elástica (dez doentes), demonstraram que este último procedimento causa elevação precoce e significativa da pressão deste esfincter.

Viazis et al. ${ }^{8}$ realizaram manometria esofágica quatro semanas após a erradicação de varizes por meio de ligadura elástica e escleroterapia em dois grupos de cirróticos e demonstraram que no grupo submetido à escleroterapia ocorreu tanto diminuição na amplitude das ondas peristálticas como aumento de contrações simultâneas, sendo que pós-ligadura não foram registradas alterações nestes parâmetros. Concluíram que a ligadura tem vantagens sobre a escleroterapia por não causar dismotilidade esofágica pós-tratamento endoscópico.

No presente estudo, como a manometria foi realizada quatro semanas após a erradicação das varizes, foi possível a avaliação dos efeitos tardios da ligadura na motilidade esofágica. Foi registrada elevação estatisticamente significativa da amplitude e da velocidade de propagação das ondas contráteis pós-ligadura. Quanto à morfologia e presença de ondas terciárias, não foram constatadas alterações antes ou após o procedimento. Foi registrado apenas um paciente que, após a ligadura, passou a apresentar ondas aperistálticas.

É importante relatar que algumas publicações mostram que a simples presença de varizes no esôfago poderia reduzir a amplitude média e aumentar a duração média das ondas peristálticas, bem como aumentar a extensão e diminuir a pressão do IIE $^{16,19}$. Este estudo e outras publicações não confirmaram estas observações ${ }^{6,8,10}$.

Alguns autores demonstraram que a ascite pode diminuir a pressão do EIE ${ }^{16}$, porém, em nosso estudo foi comparada a pressão do EIE dos doentes com e sem ascite e não foi observada nenhuma diferença.

As diferenças de relatos quanto a alterações de motilidade esofágica após procedimentos endoscópicos poderiam ser decorrentes de grupos de hepatopatas diferentes quanto à classificação de Child-Puch analisados ou eventualmente quanto ao diâmetro das varizes esofágicas consideradas. Este assunto ainda deve ser mais pesquisado. 
SOARES ACF ET AL.

\section{ConCLUSÃo}

A análise dos resultados mostrou que ocorreu alteração de motilidade esofágica pós-ligadura, caracterizada por aumento da amplitude e da velocidade de propagação das ondas contráteis.

\section{Conflito de interesse: não há.}

\section{SUMMARY}

ESOPHAGEAL MOTILITY STUDIES IN CIRRHOTIC PATIENTS BEFORE AND AFTER ENDOSCOPIC VARICEAL LIGATION

BACKGROUND. To study esophageal motility before and after the endoscopic variceal ligation in cirrhotic patients.

METHODS. Twenty-four patients with chronic liver disease assisted at the "Gastrocentro - UNICAMP" in the program of endoscopic variceal ligation were studied (mean age of 49.5 years, 19 males and 5 females). The inclusion criteria were chronic liver disease and esophageal varices with high-risk bleeding. Initially upper digestive endoscopy and esophageal manometry were performed in all cases. Next, the sessions of variceal ligation were initiated (average 3.4 \pm 2 . I) in the outpatient clinic, with an interval of 2 to 4 weeks. The esophageal manometry was repeated 4 weeks after variceal eradication and amplitude, duration, propagation speed of the contractile waves and peristalsis were studied.

RESULTS. Analysis of the lower sphincter pressure did not show differences before and after variceal ligation. In 10 cases (41.6\%) dysmotility was recorded, and the amplitude of the contractile waves rose from $70.7 \mathrm{mmHg}(52.3$ and 108.4) before variceal ligation to $89.7 \mathrm{mmHg}$ (69.9 and 122.8) after the procedure $(p=0.004-p<0.05)$, and the duration increased from $3.55 \mathrm{seg} . \pm 0.58$ to $3.90 \mathrm{seg} . \pm 0.72(p=0.02$ $p<0.05)$. The wave propagation speed did not differ from the values before the exam of $3.43 \pm 0.97 \mathrm{~cm} / \mathrm{seg}$, to those after of. $61 \pm 0.99 \mathrm{~cm} /$ $\sec (p=0.15-p>0.05)$.

CONCLUSION. The finalanalysis shows alterations of esophageal motility characterized by increase in the amplitude and duration of the contractile waves. [Rev Assoc Med Bras 2006; 52(2): 93-6]

KEY WORDS: Esophageal varices. Digestive endoscopy. Liver cirrhosis. Esophageal motility.

\section{REFERÊNCIAS}

I. Collaguazu PMC, Andreollo NA, Lopes LR, Santos JOM, Yamanaka A, Magalhães AFN, et al. Associação entre lesões de mucosa gastroduodenal e varizes como causa de hemorragia digestiva alta. Estudo retrospectivo em Hospital Universitário. Arq Gastroenterol 1993;30:88-93.

2. Andreollo NA, Miquelino M, Lopes LR, Santos JOM, Carvalho Jr AA, Montes CG, et al. Tratamento endoscópico das varizes esofágicas: ainda há lugar para a escleroterapia? GED 2002;21:59-63.
3. Rubenstein $J H$, Eisen GM, Inadomi JM. A cost-utility analysis of secondary prophylaxis for variceal hemorrhage. Am J Gastroenterol 2004;99: | 274-88.

4. Hedberg SE, Fowler DL, Ryan RLR. Injection scleroterapy of esophageal varices using ethanolamine oleato. Am J Surg 1982; | 43:426-31.

5. Larson GM, Vandertoll DJ, Netsher DT, Polk HC. Esophageal motility effects of injection sclerotherapy. Surgery 1982;96:703-9.

6. Andreollo NA, Brandalise NA, Leonardi LS. A motilidade esofágica após escleroterapia de varizes. GED 1986;5:17-21.

7. Berner JS, Gaing AA, Sharma R, Almenoff PL, Muhlfeelder T, Korsten MA. Sequelae after esophageal variceal ligation and sclerotherapy: A prospective randomized study. Am J Gastrenterol 1994;89:852-8.

8. Viazis N, Armonis A, Vlachogiannakos GR, Stefanidis G, Papadimitriou N, Manolakopoulos S, et al. Effects of endoscopic variceal treatment on esophageal function: a prospective, randomized study. Eur J Gastroenterol Hepatol 2002; 12:263-51.

9. Steigmann GV, Sun JH, Hammond WS. Results of experimental endoscopic variceal varix ligation. Am Surg 1988;54:105-8.

10. Chen S, Lo G, Lai K, Jeng J, Shen M, Huang R, et al. Endoscopic variceal ligation and sclerotherapy. J Gastroenterol Hepatol 1999; | 4:231-5.

II. Goff JS, Reveille M, Stiegmann GV. Three years experience with endoscopic variceal ligation for treatment of bleeding varices. Endoscopy | 992;24:40 |-4.

12. Greg VS, GoffJS, Michaletz-Onody PA, Korula J, Lieberman D, Saeed ZA, et al. Endoscopic sclerotherapy as compared with endoscopic ligation for beending esophageal varices. N Engl J Med 1992;236: I 527-32.

13. Goff JS, Revelle RM, Stiegmann GV. Endoscopic sclerotherapy versus endoscopic variceal ligation: esophageal symptoms, complications and motility. Am J Gastroenterol 1988;83: 1240.

14. Zargar SA, Javid G, Khan BA, Shah OJ, Yattoo GN, Shah AH, et al. Endoscopic ligation vs. sclerotherapy in adults with extrahepatic portal venous obstruction: a prospective randomized study. Gastrointest Endosc 2005;61:58-66

15. Lopes CV, Pereira-Lima JC, Pereira-Lima LF, Hornos AP, Marques DL, Cassal AP, et al. The efficacy of endoscopic ligation for the prevention of variceal rebleeding in cirrhotic patients according to the hepatocellular function. Hepatogastroenterology 2004;51:195-200.

16. Kim CH, Song JH, Kim HE, Suck CC, Lyou HL, Kim TH, et al. Effects of endoscopic variceal ligation in lower esophageal motor function: a prospective study. Korean J Int Med 1995; 10: 1 20-4.

17. Passaretti S, Mazzotti G, De Franchis R, Cipolla M, Testoni PA, Tittobello A. Esophageal motility in cirrhotics with and without esophageal varices. Scand J Gastroenterol 1989;24:334-8.

18. Avgerinos A, Viazis N, Armonis A, Vlachogiannakos J, Rekoumis G, Stefanidis $G$, et al. Early increase of lower oesophageal sphincter pressure after band ligation of oesophageal varices in cirrhotics: an intriguing phenomenon Eur J Gastroenterol Hepatol 2002 ; | 4: | 319-23.

19. Fass R, Landau O, Kovasc TOG, Ippoliti AD. Esophageal motility abnormalities in cirrhotic patients before and after endoscopic variceal treatment. Am J Gastrentorol 1997;6:94I-6.

Artigo recebido: 30/08/04 Aceito para publicação: 2 1/1 1/05 